\title{
DETECTING MAPPING SPACES
}

\author{
ALYSON BITTNER
}

\begin{abstract}
We show if $A$ is a finite $\mathrm{CW}$-complex such that algebraic theories detect mapping spaces out of $A$, then $A$ has the homology type of a wedge of spheres of the same dimension. Furthermore, if $A$ is simply connected then $A$ has the homotopy type of a wedge of spheres.
\end{abstract}

\section{INTRODUCTION}

The problem of studying the structure of mapping spaces has rich history in homotopy theory. The main goal here is to find out, for a given a space $A$, how one can characterize spaces of the homotopy type of pointed mapping spaces $\operatorname{Map}_{*}(A, Y)$. An example of a powerful result which solves this problem in a special case is the Sullivan conjecture proved by Miller in 12 . It says that $\operatorname{Map}_{*}(B \mathbb{Z} / p, Y)$ is a contractible space for all finite $\mathrm{CW}$-complexes $Y$. Another example, and one which serves as a motivation of this paper, is the description of the structure of $n$-fold loop spaces accomplished by Stasheff [15, May [11, Bordman-Vogt [5], Segal [14, Bousfield [6], and others. Their work establishes criteria for recognizing if a space $X$ has a homotopy type of a mapping space $\Omega^{n} Y=\operatorname{Map}_{*}\left(S^{n}, Y\right)$ for some $Y$, as well as methods for reconstructing the space $Y$ out of $X$. Various approaches led to different ways of expressing these results, in the language of operads, PROPs, theories, special simplicial spaces, Eilenberg-MacLane objects etc. However, as much as they are different, there is a common feature they share. In each case a space $X$ is shown to be an $n$-fold loop space if it admits certain maps between finite Cartesian products of $X$ :

$$
X^{k} \rightarrow X^{l}
$$

(or, in the case of Segal and Bousfield's work maps $X[k] \rightarrow X[l]$, where $X[k] \simeq X^{k}$, $\left.X[l] \simeq X^{l}\right)$, and if compositions of these maps satisfy appropriate relations. In effect this shows that spaces of the homotopy type of loop spaces can be thought of as a kind of algebraic objects in the category $\mathbf{S p a c e s}_{*}$ of pointed topological spaces.

Inspired by these results in this paper we investigate spaces $A$ with the property that mapping spaces $\operatorname{Map}_{*}(A, Y)$ can be described as spaces equipped with some finitary algebraic structure. To make it precise, we assume that there exists an algebraic theory $\mathbf{T}$ in the sense of Lawvere, such that the homotopy category of algebras over $\mathbf{T}$ is equivalent to the homotopy category of mapping $\operatorname{spaces}^{\operatorname{Map}_{*}}(A, X)$. As we explain in Section 2, the language of algebraic theories subsumes all formalisms used to describe loop spaces mentioned above, so existence of an operad, PROP etc. characterizing mapping spaces implies existence of an algebraic theory satisfying the above condition. If such an algebraic theory exists then we say that $A$ is a detectable space (see 2.2 for the precise definition). The results on the recognition of loop spaces imply that spheres $S^{n}$ are detectable for all $n \geq 0$, and their direct 
generalization gives that spaces of the form $\bigvee_{m} S^{n}$ are detectable for all $m, n \geq 0$. Our main result can be stated as follows:

Theorem 1.1. Let $A$ be a detectable finite pointed $C W$-complex, then

$$
H_{*}(A, \mathbb{Z}) \cong H_{*}\left(\bigvee_{m} S^{n}, \mathbb{Z}\right)
$$

for some $m, n \geq 0$.

As a consequence we obtain

Corollary 1.2. If $A$ is a simply connected finite pointed $C W$-complex then $A$ is detectable if and only if $A \simeq \bigvee_{m} S^{n}$ for some $m \geq 0, n>1$.

We note that the class of detectable spaces is larger if we consider also infinite CW-complexes. For example Sartwell [13] showed that spheres localized at a set of primes are detectable.

Organization of the paper. In section 2 we introduce algebraic theories, their algebras and describe how they can be used to detect mapping spaces. The main idea of the proof of Theorem 1.1 is to show that if a space $A$ is detectable then mapping spaces $\operatorname{Map}_{*}(A, X)$ are preserved by localization functors. In sections 3 and 4 we show that this preservation property imposes certain conditions on the space A. Lastly, in section [5] we complete the proof of Theorem 1.1

Acknowledgement. I would like to thank my advisor, Bernard Badzioch, for his guidance that led to this result, and his comments through many versions of this paper. An additional thanks to Emmanuel Dror Farjoun for the reference that inspired this work as well as some helpful conversations.

\section{Algebraic Theories}

Definition 2.1. An algebraic theory $\mathbf{T}$ is a simplicial category with objects $T_{0}, T_{1}, \ldots$ such that $T_{n}$ is the $n$-fold categorical product of $T_{1}$. In particular $T_{0}$ is the terminal object in $\mathbf{T}$. We assume that it is also the initial object.

Given an algebraic theory $\mathbf{T}$, a $\mathbf{T}$-algebra is a product-preserving simplicial func-

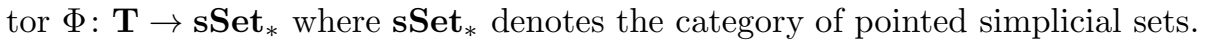

We will denote by $\mathbf{A l g}^{\mathbf{T}}$ the category of all strict $\mathbf{T}$-algebras with natural transformations of functors as morphisms. We have a forgetful functor

$$
U: \mathbf{A l g}^{\mathbf{T}} \rightarrow \mathbf{s S e t}_{*}
$$

given by $U(\Phi)=\Phi\left(T_{1}\right)$. We will say that the geometric realization of the simplicial set $U(\Phi)$ is the underlying space of the algebra $\Phi$. Intuitively, giving a $\mathbf{T}$-algebra $\Phi$ amounts to defining a certain algebraic structure, determined by $\mathbf{T}$, on the underlying space of $\Phi$.

Algebraic theories appear naturally as a tool for describing homotopy structures on spaces. Out of the formalisms characterizing loop spaces mentioned in Section 1 is the work of Boardman and Vogt explicitly uses algebraic theories. Furthermore, any operad $\mathcal{C}$ defines an algebraic theory $\mathbf{T}_{\mathcal{C}}$ as follows. Take the monad $F$ : sSet $\rightarrow$ sSet associated with the operad $\mathcal{C}$ and consider free algebras $F\left(\bigvee_{k} S^{0}\right)$ over $F$ for $k \geq 0$. These algebras define a full subcategory $\mathbf{D}$ in the category of all algebras over $F$. The algebraic theory $\mathbf{T}_{\mathcal{C}}$ is isomorphic to the opposite category of $\mathbf{D}$. One can 
check that the category of algebras over the operad $\mathcal{C}$ is isomorphic to the category of strict $\mathbf{T}_{\mathcal{C}}$-algebras. A similar reasoning can be used to construct an algebraic theory $\mathbf{T}$ associated with a given PROP in such a way, that spaces equipped with an action of the PROP coincide with spaces equipped with a structure of a strict $\mathbf{T}$ algebra. The relationship between algebraic theories and special simplicial spaces of Segal or Eilenberg-MacLane objects of Bousfield is somewhat more involved, but it has been established by Badzioch in 2 .

Algebraic theories have been used to detect mapping spaces via an equivalence between the categories of algebras and the category of topological spaces with a particular model category structure. The category of algebras $\mathbf{A l g}^{\mathbf{T}}$ over a theory $\mathbf{T}$ can be equipped with a model category structure where a natural transformation $\Phi \rightarrow \Psi$ is a weak equivalence or fibration if the induced map $\Phi\left(T_{1}\right) \rightarrow \Psi\left(T_{1}\right)$ is a weak equivalence or fibration respectively in the category of simplicial sets. Given a $C W$-complex $A$, denote by $\mathbf{R}^{A} \mathbf{T o p}$ * the category $\mathbf{T o p}_{*}$ of pointed topological spaces as a simplicial category (with $\operatorname{Hom}_{\mathbf{R}^{A}} \operatorname{Top}_{*}(X, Y)$ the simplicial mapping space $\operatorname{Sing}\left(\operatorname{Map}_{*}(X, Y)\right)$ which we will denote by $\left.\operatorname{Map}_{*}^{\Delta}(X, Y)\right)$, taken with the model category structure where fibrations are Serre fibrations and a weak equivalences are maps $f: X \rightarrow Y$ that induce weak equivalences on simplicial mapping complexes $f_{*}: \operatorname{Map}_{*}^{\Delta}(A, X) \rightarrow \operatorname{Map}_{*}^{\Delta}(A, Y)$. In other words, $\mathbf{R}^{A} \mathbf{T o p}_{*}$ is obtained by taking the right Bousfield localization of the usual model category structure on $\mathbf{T o p}_{*}$ with respect to the space $A$ as in [10, 5.1.1].

Definition 2.2. $A$ is detectable if there exists an algebraic theory $\mathbf{T}$ such that there is a Quillen equivalence

$$
\tilde{B}: \mathbf{A l g}^{\mathbf{T}} \rightleftarrows R^{A} \mathbf{T o p}_{*}: \tilde{\Omega}
$$

with underlying space $U(\tilde{\Omega} X)$ naturally weakly equivalent to $\operatorname{Map}_{*}(A, X)$.

The Quillen equivalence $(\tilde{B}, \tilde{\Omega})$ means that any mapping space $\operatorname{Map}_{*}(A, X)$ can be equipped (up to weak equivalence) with a $\mathbf{T}$-algebra structure. Moreover, if $\Phi$ is a $\mathbf{T}$-algebra, then $U(\Phi) \simeq \operatorname{Map}_{*}(A, \tilde{B} \Phi)$, so any $\mathbf{T}$-algebra comes from a mapping space.

Example 2.3. Given a pointed space $A$ there is a canonical algebraic theory $\mathbf{T}^{A}$ such that

$$
\operatorname{Hom}_{\mathbf{T}^{A}}\left(T_{n}, T_{m}\right)=\operatorname{Map}_{*}^{\Delta}\left(\bigvee^{m} A, \bigvee^{n} A\right) .
$$

The functor $\Omega^{A}: R^{A} \mathbf{T o p}_{*} \rightarrow \mathbf{A l g}^{T^{A}}$ defined by $\Omega^{A}(X)\left(T_{k}\right)=\operatorname{Map}_{*}^{\Delta}\left(\bigvee_{k} A, X\right)$ has a left adjoint $B^{A}$. The adjunction is not always a Quillen equivalence, but in particular,

$$
B^{S^{n}}: \mathbf{A l g}^{\mathbf{T}} \rightleftarrows R^{A} \mathbf{T o p}_{*}: \Omega^{S^{n}}
$$

is a Quillen equivalence [3] as well as

$$
B^{S_{P}^{n}}: \mathbf{A l g}^{\mathbf{T}} \rightleftarrows R^{A} \mathbf{T o p}_{*}: \Omega_{P}^{S_{P}^{n}}
$$

where $S_{P}^{n}$ is the sphere localized at a set of primes [13]. It follows that spheres and spheres localized at a set of primes are detectable, as well as their wedges of the same dimension. 
For our purposes it will be useful to rephrase the notion of detectability using homotopy algerbas in place of strict algebras. A homotopy algebra over an algebraic theory $\mathbf{T}$ is a simplicial functor $\mathbf{T} \rightarrow \mathbf{s S e t}_{*}$ that preserves products up to a weak equivalence. We denote the category of homotopy algebras over $\mathbf{T}$ by $\mathbf{h} \mathbf{A} \mathbf{l g}^{\mathbf{T}}$. By the rigidification theorem of algebras [1, 1.3], there is a Quillen equivalence $\mathbf{h} \mathbf{A} \mathbf{l g}^{\mathbf{T}} \rightleftarrows \mathbf{A} \lg ^{\mathbf{T}}$ between the categories of homotopy and strict algebras over $\mathbf{T}$. Using this fact, we obtain:

Proposition 2.4. A space $A$ is detectable if and only if there is some algebraic theory $\mathbf{T}$ such that there is a Quillen equivalence

$$
\tilde{B}: \mathbf{h A l g}{ }^{\mathbf{T}} \rightleftarrows R^{A} \mathbf{T o p}_{*}: \tilde{\Omega}
$$

with underlying space $U(\tilde{\Omega} X)$ weakly equivalent to $\operatorname{Map}_{*}(A, X)$

\section{L-Good Spaces}

As the first step towards proving Theorem[1.1 we will show that if $A$ is detectable, then mapping spaces out of $A$ behave like loop spaces with respect to localization functors. Let $f: Z \rightarrow W$ be a map of pointed spaces, a pointed space $X$ is said to be $f$-local if the induced map

$$
f_{*}: \operatorname{Map}_{*}(W, X) \rightarrow \operatorname{Map}_{*}(Z, X)
$$

is a homotopy equivalence. The $f$-localization of $X$, denoted $L_{f} X$, is a functorial construction of the $f$-local space closest to $X$. Explicitly, $L_{f} X$ is equipped with a coaugmentation map $X \rightarrow L_{f} X$ and given any $f$-local space $Y$ with a map $g: X \rightarrow Y$, there is a factorization unique up to homotopy:

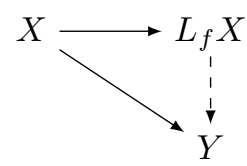

In the particular case where $f: Z \rightarrow *$ is a constant map, we denote $L_{f} X$ by $P_{Z} X$ and call it the $Z$-nullification of $X$. If $X$ is local with respect to the map $Z \rightarrow *$ that is, $\operatorname{Map}_{*}(Z, X) \simeq *$ we say $X$ is a $Z$-null space.

It is well known [9, 3.A.1] that loop spaces commute with localization functors in the sense that for any map $f$ of pointed spaces and $X$ a pointed space there is a weak equivalence

$$
L_{f} \Omega X \simeq \Omega L_{\Sigma f} X .
$$

This property is generalized with the following definition.

Definition 3.1. A space $A$ is $L$-good if for any map $f$ the localization functor $L_{f}$ preserves mapping spaces out of $A$, that is,

$$
L_{f} \operatorname{Map}_{*}(A, X) \simeq \operatorname{Map}_{*}(A, Y) .
$$

Proposition 3.2. If the space $A$ is detectable, then $A$ is an L-good space.

Proof. Assume $A$ is detectable by the algebraic theory $\mathbf{T}$ and let $X$ be any space. Then $\operatorname{Map}_{*}(A, X)$ is weakly equivalent to the underlying space of some algebra $\Phi$ over T. $L_{f}$ preserves products up to a weak equivalence, so $L_{f} \circ \Phi$ is a homotopy algebra with underlying space $L_{f} \operatorname{Map}_{*}(A, X)$. By detectability of $A$, the underlying 
space of any homotopy algebra over $\mathbf{T}$, in particular $L_{f} \circ \Phi$, is up to weak equivalence a mapping space out of $A$. We then obtain

$$
L_{f} \operatorname{Map}_{*}(A, X) \simeq \operatorname{Map}_{*}(A, Y)
$$

for some space $Y$.

If $A$ is an $L$-good finite $\mathrm{CW}$-complex, then the rational homotopy type of $A$ is determined.

Theorem 3.3. [4, 1.2] Let $A$ be a finite, connected, pointed $C W$-complex such that for some $p>q>0$ we have $H^{p}(A, \mathbb{Q}) \neq 0 \neq H^{q}(A, \mathbb{Q})$. Then $A$ is not an L-good space.

This result can be extended to rule out some cases of torsion.

Proposition 3.4. Let $A$ be a finite, pointed, connected $C W$-complex. If $A$ is $L$-good and $H_{n}(A, \mathbb{Z})$ contains a torsion-free element for some $n>0$ then $H_{q}(A, \mathbb{Z})=0$ for all $0<q<n$.

In order to prove this fact, we need to introduce the concept of celluarization, a generalization of $\mathrm{CW}$-approximation. We say that a space $X$ is $A$-cellular if $X$ is in the smallest class containing $A$ which is closed under homotopy colimits and weak equivalences. The space $C W_{A} X$ associated to a space $X$ is the $A$-cellular space closest to $X$. More precisely, we say that a map $f: X \rightarrow Y$ is an $A$-equivalence if the induced map

$$
\operatorname{Map}_{*}(A, X) \rightarrow \operatorname{Map}_{*}(A, Y)
$$

is a homotopy equivalence. Given a space $X$, the $A$-cellular approximation of $X$ is an $A$ cellular space $C W_{A} X$ equipped with an $A$-equivalence $X \rightarrow C W_{A} X$. The assignment $X \mapsto C W_{A} X$ defines a functor $C W_{A}: \mathbf{S p a c e s}_{*} \rightarrow \mathbf{S p a c e s}_{*}$ [9, 2.B].

Given any map $Y \rightarrow X$ where $Y$ is $A$-cellular there is a factorization

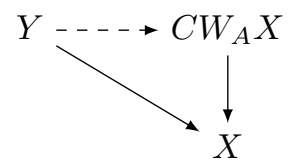

unique up to homotopy. The following theorem describes the relationship between cellularization and nullification.

Theorem 3.5. 9, 3.B.2] If $[A, X] \simeq *$, then the sequence

$$
C W_{A} X \rightarrow X \rightarrow P_{\Sigma A} X
$$

is a fibration sequence.

Recall that Generalized Eilenberg-Maclane spaces (GEMs) are spaces weakly equivalent to a product of Eilenberg-Maclane spaces $\tilde{\Pi}_{n} K\left(G_{n}, n\right)$. Localization and cellularization preserves GEMs in particular cases.

Lemma 3.6. 9, 5.B.1.1] If $P_{\Sigma A} X \simeq *$ then $P_{\Sigma^{2} A} X$ is a GEM.

Lemma 3.7. Let $A, X$ be connected spaces such that $\operatorname{Map}_{*}(A, X) \simeq K(G, 1)$ for some group $G$, then $C W_{A}(X)$ is a GEM. 
Proof. Since $\left[A, C W_{A} X\right] \cong[A, X]=\pi_{0}\left(\operatorname{Map}_{*}(A, X)\right)=*$, by Theorem 3.5 we have a fibration sequence

$$
C W_{A} C W_{A} X \rightarrow C W_{A} X \rightarrow P_{\Sigma A} C W_{A} X .
$$

The map $C W_{A} C W_{A} X \rightarrow C W_{A} X$ is a weak equivalence, which gives $P_{\Sigma A} C W_{A} X \simeq$ * and by Lemma 3.6 $P_{\Sigma^{2}{ }_{A}} C W_{A} X$ is a GEM. It then suffices to show $C W_{A} X \simeq$ $P_{\Sigma^{2} A} C W_{A} X$, or equivalently that $C W_{A} X$ is a $\Sigma^{2} A$-null space. This holds since

$$
\operatorname{Map}_{*}\left(\Sigma^{2} A, C W_{A} X\right) \simeq \Omega^{2} \operatorname{Map}_{*}(A, X) \simeq \Omega^{2} K(G, 1) \simeq * .
$$

Proof of Proposition 3.4. Assume that $A$ is a finite, pointed, connected $C W$-complex such that $H_{n}(A, \mathbb{Z})$ contains a torsion-free element and that there exists $0<m<n$ such that $H_{m}(A, \mathbb{Z}) \neq 0$. For the sake of contradiction, suppose $A$ is $L$-good, then by Theorem $3.3 . H_{m}(A ; \mathbb{Z})$ must be torsion.

Consider the map $f: S^{2} \vee\left(\bigvee_{p \in P} S^{1}\right) \rightarrow\left(\bigvee_{p \in P} S^{1}\right)$, where $P$ is the set of all primes, such that $\left.f\right|_{S^{2}}=*$ and $\left.f\right|_{S_{p}^{1}}: S_{p}^{1} \rightarrow S_{p}^{1}$ is the degree $p$ map $S^{1} \rightarrow S^{1}$. The localization $L_{f} X$ of a space $X$ has trivial homotopy groups $\pi_{i}\left(L_{f} X\right)$ for $i>1$ and has no torsion in the fundamental group and so

$$
L_{f} X=K\left(\pi_{1}(X) / \operatorname{Tor}\left(\pi_{1}(X)\right), 1\right) .
$$

We will show that $A$ is not $L$-good with respect to the map $f$. Indeed, otherwise there would exist a space $Y$ (which can be assumed to be $A$-cellular) such that

$$
L_{f} \operatorname{Map}_{*}(A, K(\mathbb{Z}, n+1)) \simeq \operatorname{Map}_{*}(A, Y) .
$$

However, $L_{f} \operatorname{Map}_{*}(A, K(\mathbb{Z}, n+1)) \simeq K\left(H^{n}(A ; \mathbb{Z}) / \operatorname{Tor}\left(H^{n}(A ; \mathbb{Z})\right), 1\right)$. By assumption, $H_{n}(A, \mathbb{Z})$ has a nontrivial torsion-free part so $H_{n}(A, \mathbb{Z}) \cong \mathbb{Z}^{k} \oplus \operatorname{Tor}\left(H_{n}(A, \mathbb{Z})\right)$ for some $k>0$. By the universal coefficient theorem, the torsion-free part of $H^{n}(A ; \mathbb{Z})$ is isomorphic to the torsion-free part of $H_{n}(A, \mathbb{Z})$. This gives

$$
\operatorname{Map}_{*}(A, Y) \simeq K\left(\mathbb{Z}^{k}, 1\right) \text {. }
$$

By Lemma 3.7 we obtain, $Y \simeq \tilde{\Pi}_{i=1}^{\infty} K\left(G_{i}, i\right)$ and so

$$
\operatorname{Map}_{*}\left(A, \tilde{\Pi}_{i} K\left(G_{i}, i\right)\right) \simeq K\left(\mathbb{Z}^{k}, 1\right) .
$$

Restricting our attention to the fundamental groups, we get $\oplus_{i} \tilde{H}^{i-1}\left(A, G_{i}\right) \cong \mathbb{Z}^{k}$ where each of the summands on the left side is torsion, hence trivial, with the exception of $i=n+1$. We obtain

$$
\mathbb{Z}^{k} \cong H^{n}\left(A ; G_{n+1}\right) \cong \operatorname{Hom}\left(H_{n}(A, \mathbb{Z}), G_{n+1}\right) \oplus \operatorname{Ext}\left(H_{n-1}(A, \mathbb{Z}), G_{n+1}\right)
$$

which implies

$$
\mathbb{Z}^{k} \cong \operatorname{Hom}\left(H_{n}(A, \mathbb{Z}), G_{n+1}\right)
$$

as $\operatorname{Ext}\left(H_{n-1}(A, \mathbb{Z}), G_{n+1}\right)$ is torsion. Note that $\operatorname{Hom}\left(H_{n}(A, \mathbb{Z}), G_{n+1}\right)=\operatorname{Hom}\left(\mathbb{Z}^{k} \oplus\right.$ $\left.\operatorname{Tor}\left(H_{n}(A, \mathbb{Z})\right), G_{n+1}\right)$ and so $\mathbb{Z}^{k} \cong \bigoplus_{i=1}^{k} G_{n+1} \oplus$ (torsion) which implies $G_{n+1} \cong \mathbb{Z}$.

Now, $H_{m}(A, \mathbb{Z})$ is torsion, so by the universal coefficient theorem it is a direct summand of $H^{m+1}(A, \mathbb{Z})$ so we have

$$
H_{m}(A, \mathbb{Z}) \subseteq H^{m+1}(A, \mathbb{Z}) \cong \pi_{n-m} \operatorname{Map}_{*}(A, K(\mathbb{Z}, n+1)) .
$$

Since $G_{n+1} \cong \mathbb{Z}$, the space $\operatorname{Map}_{*}(A, K(\mathbb{Z}, n+1))$ is a retract of $\operatorname{Map}_{*}(A, Y)$ and so

$$
H_{m}(A, \mathbb{Z}) \subseteq \pi_{n-m}\left(\operatorname{Map}_{*}(A, Y)\right)=\pi_{n-m}\left(K\left(\mathbb{Z}^{k}, 1\right)\right)
$$


which is a contradiction. This shows that the space $Y$ cannot exist and so $A$ is not L-good.

Proposition 3.8. Let $A$ be a finite, pointed, connected $C W$-complex. If $H_{n}(A, \mathbb{Z}) \cong$ $\mathbb{Z}^{k} \oplus T$ for $k>0$ and $T$ is a non-trivial torsion group, then $A$ is not L-good.

Proof. We can use the same argument as the previous proposition to show that if $f$ is the map as in the proof of Proposition 3.4 and $L_{f} \operatorname{Map}_{*}(A, K(\mathbb{Z}, n+1)) \simeq$ $\operatorname{Map}_{*}(A, Y)$ then

$$
T \subseteq \pi_{0} \operatorname{Map}_{*}(A, K(\mathbb{Z}, n+1)) \subseteq \pi_{0}\left(\operatorname{Map}_{*}(A, Y)\right)=\pi_{0}\left(K\left(\mathbb{Z}^{k}, 1\right)\right)
$$

which is a contradiction.

Corollary 3.9. If $A$ is a finite, pointed, connected, $C W$-complex that is $L$-good and $H_{n}(A, \mathbb{Z})$ contains a torsion-free element for some $n>0$, then

$$
H_{q}(A ; \mathbb{Z}) \cong\left\{\begin{array}{cc}
0 & \text { if } 0<q<n \\
\mathbb{Z}^{k} & \text { if } q=n \\
\text { torsion } & \text { if } q>n
\end{array}\right.
$$

\section{Commutation of Localization}

By definition, if $A$ is an $L$-good space then the class of mapping $\operatorname{spaces}^{\operatorname{Map}_{*}}(A, X)$ is preserved by localization functors. If $A=S^{n}$, the homotopy type of the space $L_{f} \operatorname{Map}_{*}(A, X)$ can be described more precisely as follows:

Theorem 4.1. [7, 3.1] Let $f: Z \rightarrow W$ be any map in Spaces $_{*}$ and $X$ a pointed space. The natural map

$$
L_{f} \operatorname{Map}_{*}\left(S^{n}, X\right) \rightarrow \operatorname{Map}_{*}\left(S^{n}, L_{S^{n} \wedge f} X\right)
$$

is a homotopy equivalence.

This result motivates the following definition:

Definition 4.2. A space $A$ is strongly $L$-good if for any space $X$ and any map $f$, there exists a map $g$ such that

$$
L_{f}\left(\operatorname{Map}_{*}(A, X)\right) \simeq \operatorname{Map}_{*}\left(A, L_{g}(X)\right) .
$$

We will show that the following holds:

Theorem 4.3. If $A$ is a finite $C W$ complex that is strongly $L$-good, then

$$
H_{*}(A) \cong H_{*}\left(\bigvee_{l} S^{n}\right)
$$

for some $n \geq 1, l \geq 0$.

We will be utilize the following property of localizations of Eilenberg-Maclane Spaces.

Proposition 4.4. [8, 3.1] Let $f$ be any map and $n \geq 1$, then

$$
L_{f}(K(\mathbb{Z}, n)) \simeq K(G, n)
$$

for some abelian group $G$. 
Proof of Theorem 4.3. We will argue by contradiction. Assume that $A$ is strongly $L$-good, then by Corollary 3.9, we have

$$
H_{q}(A) \cong\left\{\begin{array}{cc}
0 & \text { if } 0<q<n \\
\mathbb{Z}^{l} & \text { if } q=n \\
\text { torsion } & \text { if } q>n
\end{array} .\right.
$$

Assume that $H_{q}(A) \neq 0$ for some $q>n$ and let $m>n$ be the largest integer such that $H_{m}(A) \neq 0$.

By the universal coefficients theorem, we get

$$
\pi_{i} \operatorname{Map}_{*}(A, K(\mathbb{Z}, n+m+1)) \cong \operatorname{Hom}\left(H_{n+m+1-i}(A) ; \mathbb{Z}\right) \oplus \operatorname{Ext}\left(H_{n+m-i}(A), \mathbb{Z}\right)
$$

and our assumptions on the homology of $A$ give

$$
\pi_{i}\left(\operatorname{Map}_{*}(A, K(\mathbb{Z}, n+m+1))=\left\{\begin{array}{cc}
0 & i<n \\
H_{m}(A) & i=n \\
\text { torsion } & n<i<m+1 \\
\mathbb{Z}^{l} & i=m+1 \\
0 & i>m
\end{array}\right.\right.
$$

. Let $P_{S^{n+1}}$ be the $S^{n+1}$ nullification functor, we have

$$
\pi_{i}\left(P_{S^{n+1}} \operatorname{Map}_{*}(A, K(\mathbb{Z}, n+m+1))=\left\{\begin{array}{cc}
0 & \text { otherwise } \\
H_{m}(A) & i=n \\
0 & i>m
\end{array}\right.\right.
$$

and hence $P_{S^{n+1}} \operatorname{Map}_{*}(A, K(\mathbb{Z}, n+m+1)) \simeq K\left(H_{m}(A), n\right)$. By the assumptions on $A$ we have

$$
P_{S^{n+1}} \operatorname{Map}_{*}(A, K(\mathbb{Z}, n+m+1)) \simeq \operatorname{Map}_{*}\left(A, L_{g}(K(\mathbb{Z}, n+m+1))\right) .
$$

Applying Proposition 4.4, the localization of an Eilenberg-Maclane space is again an Eilenberg-Maclane space, so we obtain

$$
\operatorname{Map}_{*}\left(A, L_{g}(K(\mathbb{Z}, n+m+1))\right) \simeq \operatorname{Map}_{*}(A, K(G, n+m+1))
$$

for some group $G$. This gives

$$
K\left(H_{m}(A), n\right) \simeq \operatorname{Map}_{*}(A, K(G, n+m+1)) .
$$

We obtain

$$
0 \cong \pi_{m+1}\left(K\left(H_{m}(A), n\right)\right) \cong H^{n}(A ; G) \cong \operatorname{Hom}\left(H_{n}(A), G\right) \oplus \operatorname{Ext}\left(H_{n-1}(A), G\right)
$$

Since $H_{n}(A)$ is a free abelian group this implies that $G$ is trivial, so

$$
K\left(H_{m}(A), n\right) \simeq \operatorname{Map}_{*}(A, K(G, n+m+1)) \simeq *
$$

which is a contradiction.

Corollary 4.5. If $A$ is a simply connected finite $C W$-complex that is strongly $L$ good space, then $A$ has the homotopy type of a wedge of spheres $\bigvee_{l} S^{k}$ for some $k>0, l>0$.

Proof. By the Hurewicz isomorphism $h: \pi_{n} X \rightarrow H_{n} X \cong \mathbb{Z}^{l}$, we have maps $\alpha_{i}$ : $S^{n} \rightarrow X$ for $i=1, \ldots, l$ such that $\left\{h\left(\alpha_{i}\right) \mid i=1, \ldots, l\right\}$ are generators of $H_{n}(X)$. The map $\bigvee_{i} \alpha_{i}: \bigvee_{i} S^{n} \rightarrow X$ induces isomorphisms of homology groups, and by the Whitehead theorem, it is a homotopy equivalence. 


\section{Proof of the Main Theorem}

In view of Theorem 4.3 in order to complete the proof of Theorem 1.1 it will suffice to show that the following holds:

Theorem 5.1. If $A$ is a detectable, finite $C W$-complex then $A$ is strongly $L$-good.

Our proof will parallel Bousfield's argument showing that loop spaces commute with localization functors (Theorem 4.1). The argument relies on Segal's machinery to detect loop spaces which we will substitute with the Quillen equivalence $(\tilde{B}, \tilde{\Omega})$ given in the definition of a detectable space (Definition 2.2. Proposition ). The Quillen equivalence descends to a derived equivalence on homotopy categories. We will choose to work within the homotopy category, and will use the following lemma.

Lemma 5.2. If $F, G: H o C \rightarrow H o D$ are coaugmented, idempotent functors such that $c \cong F(C)$ if and only if $c \cong G(c)$ then $F$ and $G$ are naturally isomorphic.

Proof. For any $c \in \mathbf{C}$ we have the following diagram.

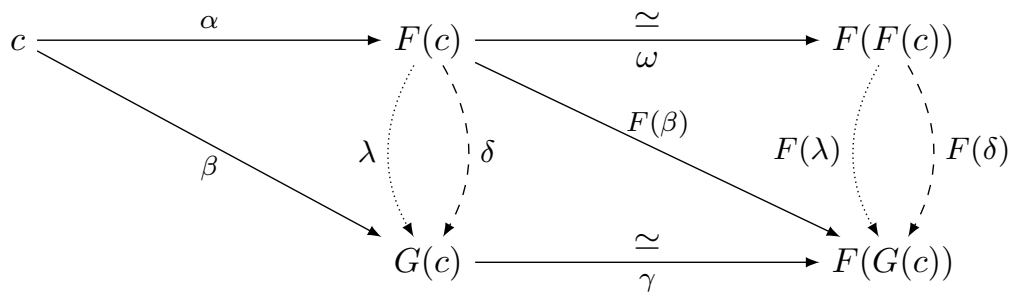

where $\alpha, \beta, \gamma$, and $\omega$ are defined by the coaugmentation maps. Since $F(c) \simeq$ $F(F(c)), G(c) \simeq F(G(c))$ and $\gamma$ is an isomorphism. We define $\delta=\gamma^{-1} \circ F(\beta)$. We first claim that $\delta$ is unique up to weak equivalence, to show this assume $\lambda$ is another map $F(c) \rightarrow G(c)$. Then we get $F(\lambda) \circ \omega \simeq F(\beta) \simeq F(\delta) \circ \omega$ and since $\omega$ is a weak equivalence we have $F(\lambda) \simeq F(\delta)$ and hence $\lambda \simeq \delta$. The factorization of $\beta$ through $\alpha$ is unique up to homotopy. We can similarly show the factorization of $\alpha$ through $\beta$ is unique up to homotopy and thus proving $\delta$ is an isomorphism.

We will use the following fact which is a direct consequence of the mapping space smash product adjunction.

Lemma 5.3. $X$ is $(A \wedge f)$-local if and only if $\operatorname{Map}_{*}(A, X)$ is $f$-local.

Proof of Theorem 5.1. Since $A$ is detectable, there is a derived equivalence on the level of homotopy categories.

$$
\tilde{B}_{\mathrm{der}}: \operatorname{HoAlg}^{T} \rightleftarrows \operatorname{Ho} R^{A} \mathbf{T o p}_{*}: \tilde{\Omega}_{\mathrm{der}} .
$$

We first claim the functors $\tilde{B}_{\mathrm{der}} L_{f} \tilde{\Omega}_{\mathrm{der}}$ and $L_{A \wedge f}$ are both coaugmented, idempotent functors with the same images, thus by lemma 5.2 are the naturally isomorphic. We have natural maps

$$
X \stackrel{\simeq}{\longleftarrow} \tilde{B}_{\mathrm{der}} \tilde{\Omega}_{\mathrm{der}} X \longrightarrow \tilde{B}_{\mathrm{der}} L_{f} \tilde{\Omega}_{\mathrm{der}} X
$$

where the latter is induced from the coaugmentation map of $L_{f}$, hence $\tilde{B}_{\mathrm{der}} L_{f} \tilde{\Omega}_{\mathrm{der}}$ is coaugmented.

We now show for $X$ in $R^{A} \mathbf{T o p}_{*}, \tilde{B}_{\mathrm{der}} L_{f} \tilde{\Omega}_{\mathrm{der}} X \simeq X$ if and only if $L_{A \wedge f} X \simeq X$. As $X \simeq \tilde{B}_{\mathrm{der}} \tilde{\Omega}_{\mathrm{der}} X$, the spaces $\tilde{B}_{\mathrm{der}} L_{f} \tilde{\Omega}_{\mathrm{der}} X$ and $X$ are weakly equivalent if and 
only if there is a weak equivalence of algebras $L_{f} \tilde{\Omega}_{\mathrm{der}} X \simeq \tilde{\Omega}_{\mathrm{der}} X$. By the model category structure on the category of algebras over a theory, $L_{f} \tilde{\Omega}_{\mathrm{der}} X \simeq \tilde{\Omega}_{\mathrm{der}} X$ if and only if the underlying spaces $L_{f} \operatorname{Map}_{*}(A, X)$ and $\operatorname{Map}_{*}(A, X)$ are weakly equivalent which implies $\operatorname{Map}_{*}(A, X)$ must be $f$-local. By lemma [5.3, we obtain that $\tilde{B}_{\mathrm{der}} L_{f} \tilde{\Omega}_{\mathrm{der}} X \simeq X$ if and only if $L_{A \wedge f} X \simeq X$.

The functors $\tilde{B}_{\mathrm{der}} L_{f} \tilde{\Omega}_{\mathrm{der}}$ and $L_{A \wedge f}$ are then naturally isomorphic, so by composing with $\tilde{\Omega}_{\mathrm{der}}$, the following algebras are weakly equivalent

$$
L_{f} \tilde{\Omega}_{\mathrm{der}} X \simeq \tilde{\Omega}_{\mathrm{der}} \tilde{B}_{\mathrm{der}} L_{f} \tilde{\Omega}_{\mathrm{der}} X \simeq \tilde{\Omega}_{\mathrm{der}} L_{A \wedge f} X
$$

with associated underlying spaces

$$
L_{f} \operatorname{Map}_{*}(A, X) \simeq \operatorname{Map}_{*}\left(A, L_{A \wedge f} X\right)
$$

completing the proof that $A$ is strongly $L$-good.

\section{REFERENCES}

[1] Bernard Badzioch. Algebraic theories in homotopy theory. Annals of mathematics, 155(3):895-913, 2002.

[2] Bernard Badzioch. From -spaces to algebraic theories. Transactions of the American Mathematical Society, 357(5):1779-1799, 2005.

[3] Bernard Badzioch, Kuerak Chung, and Alexander A Voronov. The canonical delooping machine. Journal of Pure and Applied Algebra, 208(2):531-540, 2007.

[4] Bernard Badzioch and Wojciech Dorabiała. A note on localizations of mapping spaces. Israel Journal of Mathematics, 177(1):441-444, 2010.

[5] J.M. Boardman and R.M. Vogt. Homotopy invariant algebraic structures on topological spaces. Springer, 1973.

[6] A. K. Bousfield. The simplicial homotopy theory of iterated loop spaces. Manuscript, 1992.

[7] A. K. Bousfield. Localization and periodicity in unstable homotopy theory. Journal of the American Mathematical Society, 7(4):831-873, 1994.

[8] Carles Casacuberta, José L Rodrıguez, and Jin-Yen Tai. Localizations of abelian eilenbergmac lane spaces of finite type. preprint, 1998.

[9] Emmanuel D. Farjoun. Cellular Spaces, Null Spaces and Homotopy Localization. Springer, 1995.

[10] Philip S Hirschhorn. Model Categories and Their Localizations. Number 99. American Mathematical Soc., 2009.

[11] J Peter May. The geometry of iterated loop spaces. Springer Berlin, 1972.

[12] Haynes Miller. The sullivan conjecture on maps from classifying spaces. Annals of Mathematics, 120(1):39-87, 1984.

[13] Matthew Sartwell. Detecting Mapping Spaces and Derived Equivalences of Algebraic Theories. PhD thesis, University at Buffalo, 2016.

[14] Graeme Segal. Categories and cohomology theories. Topology, 13(3):293-312, 1974.

[15] James Dillon Stasheff. Homotopy associativity of h-spaces. i. Transactions of the American Mathematical Society, 108(2):275-292, 1963. 